OPEN ACCESS

Edited by:

Zhenzhen Liu,

Sun Yat-sen University, China

Reviewed by:

Miguel Ángel Aloy,

University of Valencia, Spain

Weicong Lu,

Qingdao Eye Hospital, China

${ }^{*}$ Correspondence:

Geng Wang

wg@jsiec.org

orcid.org/0000-0001-8476-1955

Specialty section:

This article was submitted to

Ophthalmology,

a section of the journal

Frontiers in Medicine

Received: 30 June 2021 Accepted: 25 November 2021

Published: 04 January 2022

Citation:

Gong W, Chen H, Yang F, Lin S, Li C

and Wang $G$ (2022) Inter-eye

Differences in Ocular Biometric Parameters of Concomitant Exotropia.

Front. Med. 8:724122.

doi: 10.3389/fmed.2021.724122

\section{Inter-eye Differences in Ocular Biometric Parameters of Concomitant Exotropia}

\author{
Weifen Gong, Haoyu Chen, Fan Yang, Shibin Lin, Chao Li and Geng Wang* \\ Joint Shantou International Eye Center of Shantou University and The Chinese University of Hong Kong, Shantou, China
}

Purpose: To evaluate the ocular biometric parameters in patients with constant and intermittent exotropia by the measurement of swept-source optical coherence tomography (SS-OCT) optical biometer OA-2000 and comparing it with the normal control subjects.

Design: Cross-sectional case-control study.

Participants: Fifty-five constant and 24 intermittent patients with exotropia with central fixation and 77 orthotropic normal control participants aged $4-18$ years old.

Methods: Non-contact and high-resolution optical biometric OA-2000 measurements were conducted under uniform ambient light conditions. The statistical analysis included intraclass correlation coefficient (ICC), Bland-Altman plot, and independent $t$-tests.

Main Outcome Measures: Spherical equivalent (SE), ocular biological parameters such as pupil diameter (PD), anterior chamber depth (ACD), lens thickness (LT), and axial lengths (AL). The absolute values of inter-eye differences for SE, PD, ACD, LT, and AL were recorded as AnisoSE, AnisoPD, AnisoACD, AnisoLT, and AnisoAL, respectively.

Results: AnisoSE (0.878 vs. 0.577, $P=0.019$ ), AnisoAL (0.395 vs. 0.208, $P=0.001$ ), AnisoACD (0.060 vs. 0.032, $P<0.001$ ), AnisoLT (0.060 vs. $0.031, P=0.002$ ), and AnisoPD (0.557 vs. 0.340, $P=0.002)$ were significantly larger in concomitant patients with exotropia. The SE, AL, ACD, LT, and PD showed excellent binocular correlation with ICC values that ranged from 0.943 to 0.987 in control participants and from 0.767 to 0.955 in concomitant exotropia patients. Bland-Altman plots showed the wider range of agreement in patients with concomitant exotropia than the control participants (SE: 5.0288 vs. 3.3258; AL: 2.2146 vs. $1.3172 ;$ ACD:0.3243 vs. 0.1682; PD: 2.4691 vs. 1.9241; and LT:0.3723 vs 0.1858).

Conclusion: Patients with concomitant exotropia showed larger inter-eye differences in SE, ACD, LT, PD, and AL. Advice should be given to suspicious children to avoid or delay the development of concomitant exotropia.

Keywords: concomitant exotropia, ocular biometric parameters, OA-2000, intereye differences, anisometropia 


\section{INTRODUCTION}

Concomitant exotropia is a manifest divergent strabismus, defined as the deviating angle independent of gaze direction. It affects $\sim 4 \%$ of the adult population (1) and could lead to the loss of binocularity and stereopsis. Besides the functional effects, patients with concomitant exotropia experience significant psychological stress, anxiety, and depression $(2,3)$. Concomitant exotropia often negatively impacts self-esteem, self-confidence, and interpersonal relationships $(4,5)$. Some patients assume adaptive techniques to hide exotropia, such as placing their hair over the deviating eye (2). Adults with strabismus often have reduced quality of life (6), lower education levels, and fewer career choices (7).

The cause of concomitant exotropia is still controversial. The reported risk factors included maternal smoking during pregnancy (8), premature birth, perinatal morbidity, genetic anomalies (9), family history, anisometropia (10,11), and myopia (12). Some studies $(13,14)$ have reported the contributions of rectus extraocular muscles, and revealed the strong relationship between extraocular muscle's pulley position and refraction. Refractive errors, especially myopia and anisometropia, have a strong correlation with ocular biometric parameters, especially in axial length (15-18). Yet, the correlation between ocular biometric parameters and concomitant exotropia remains unclear, and few studies have evaluated the ocular biometric parameters in concomitant exotropia.
Ocular biometric measurements have been widely used in clinical practices such as the calculation of intraocular lens (IOL) power (19), candidate screening for refractive surgery, monitoring of the ametropic progression (20), estimation of the ocular mechanical properties (21), measurement of ocular anatomic changes on different accommodative stimuli $(22,23)$, or wearing contact lenses (24). As a novel non-contact and highresolution optical biometric device, OA-2000 (Tomey, Nagoya, Japan) incorporates swept-source optical coherence tomography and a Placido-disc topographer, which could automatically find a measurable point and complete the scans quickly and accurately. It shows high repeatability and reproducibility, and an excellent agreement with other optical biometric devices, such as IOL Master700 (Carl Zeiss Meditec AG, Jena, Germany) and LenstarLS900 (Haag Streit AG, Koeniz, Switzerland) (18, 20, 25, 26). In this study, we aimed to evaluate the ocular biometric parameters in patients with constant and intermittent exotropia by OA2000 and compare this with the normal control subjects to determine the contribution of ocular biometric parameters in concomitant exotropia.

\section{METHODS}

\section{Study Subjects}

The study protocol conformed to the Declaration of Helsinki and was approved by the Human Medical Research Ethics Committee of Joint Shantou International Eye Center of Shantou

TABLE 1 | Comparisons of the absolute values of inter-eye differences between concomitant exotropia and control participants.

\begin{tabular}{|c|c|c|c|c|}
\hline & XT mean (SD) & NCP mean (SD) & $T$-value ( $P$-value) & Mann-Whitney $U$-test \\
\hline AnisoSE (D) & $0.878(0.931)$ & $0.577(0.625)$ & $2.369\left(0.019^{\star}\right)$ & 0.034 \\
\hline AnisoAL (mm) & $0.395(0.404)$ & $0.208(0.265)$ & $3.423\left(0.001^{\star}\right)$ & $<0.001$ \\
\hline AnisoACD (mm) & 0.060 (0.059) & $0.032(0.030)$ & $3.782\left(<0.001^{\star}\right)$ & 0.001 \\
\hline AnisoPD (mm) & $0.557(0.476)$ & $0.340(0.357)$ & $3.229\left(0.002^{\star}\right)$ & 0.001 \\
\hline AnisoLT (mm) & 0.060 (0.073) & $0.031(0.035)$ & $3.136\left(0.002^{\star}\right)$ & 0.003 \\
\hline
\end{tabular}

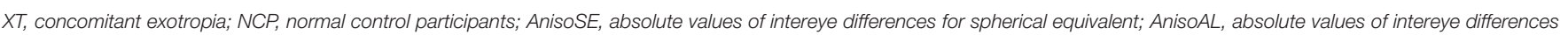

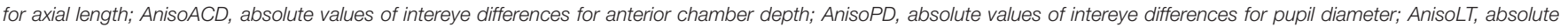
values of intereye differences for lens thickness.

${ }^{*}$ Results with equal variances not assumed and reanalyzed with Mann-Whitney U-test.

TABLE 2 | Correlation coefficients of the ocular biometric parameters between the two eyes within concomitant exotropia and control participants.

\begin{tabular}{|c|c|c|c|c|c|c|}
\hline & \multicolumn{3}{|c|}{ XT } & \multicolumn{3}{|c|}{ NCP } \\
\hline & $\begin{array}{c}\text { ICC } \\
\text { (P-value) }\end{array}$ & $\begin{array}{l}\text { Pearson's } \\
\text { correlation }\end{array}$ & $\begin{array}{c}\text { Pearson's } \\
\text { correlation P }\end{array}$ & $\begin{array}{c}\text { ICC } \\
\text { (P-value) }\end{array}$ & $\begin{array}{l}\text { Pearson's } \\
\text { correlation }\end{array}$ & $\begin{array}{c}\text { Pearson's } \\
\text { correlation P }\end{array}$ \\
\hline SE & 0.877 & 0.876 & $<0.001$ & 0.943 & 0.944 & $<0.001$ \\
\hline$A L$ & 0.914 & 0.915 & $<0.001$ & 0.971 & 0.972 & $<0.001$ \\
\hline ACD & 0.955 & 0.956 & $<0.001$ & 0.987 & 0.987 & $<0.001$ \\
\hline PD & 0.767 & 0.826 & $<0.001$ & 0.944 & 0.945 & $<0.001$ \\
\hline LT & 0.924 & 0.924 & $<0.001$ & 0.972 & 0.974 & $<0.001$ \\
\hline
\end{tabular}

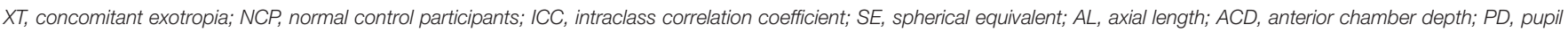
diameter; $L T$, lens thickness. 
A

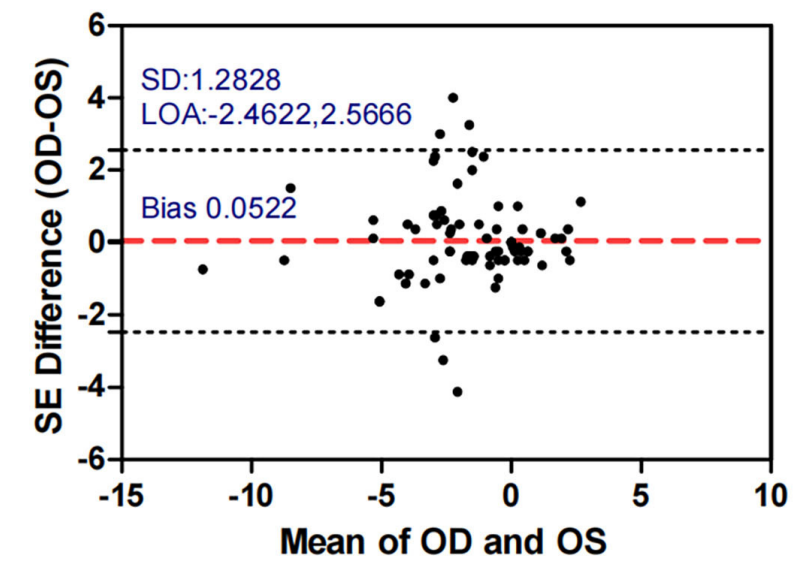

C

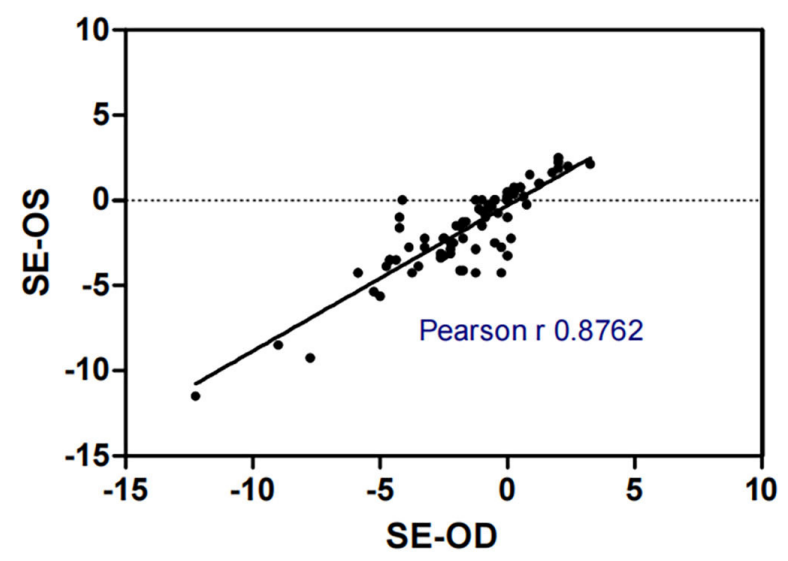

B
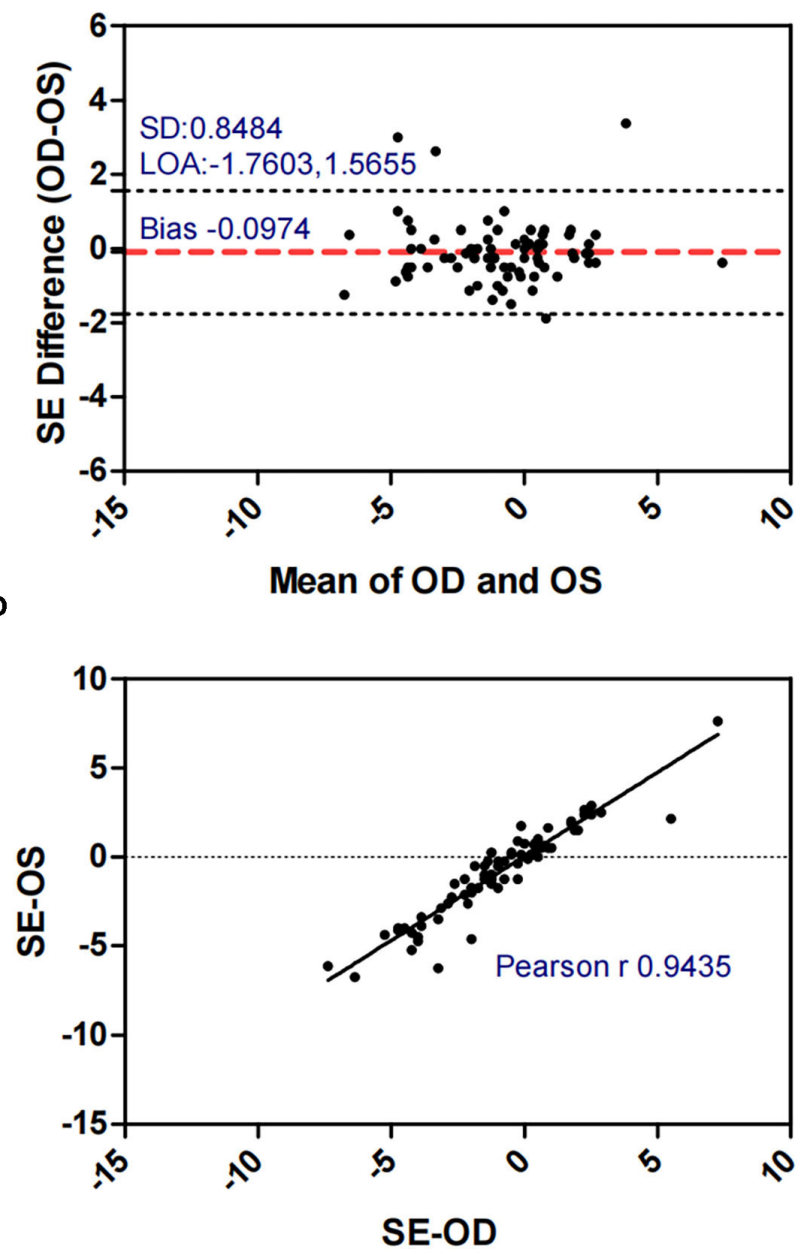

FIGURE 1 | Bland-Altman plots (A,B) and scatter plots (C,D) of SE in concomitant exotropia (A,C) and normal control participants $(\mathbf{B}, \mathbf{D})$ of the left and right eyes. OS, left eye; OD, right eye; SE, spherical equivalent; LOA, 95\% limits of agreement.

University and by the Chinese University of Hong Kong. Informed consent was obtained from the legal representatives of all subjects after a full explanation of the purpose of the study had been fully accomplished. Patients with concomitant exotropia aged 4-18 years old were recruited between July and August 2019. All subjects underwent comprehensive ophthalmic examinations, including the measurements of visual acuity, intraocular pressure, cycloplegic refraction, anterior segment, and fundus examination, corneal light reflex tests, prism alternate cover test (PACT) for ocular alignment measurement, fixation behavior, and OA-2000 for ocular biometric parameter collection. A total of 79 patients, consisting of 55 constant and 24 intermittent concomitant exotropia with central fixation, were recruited. Among the 24 intermittent patients, 10 patients had reduced stereopsis tested by Titmus, 800 arc in six patients, and 400 arc in four patients. All other patients had lost stereopsis. A total of 77 control subjects were recruited with normal ocular motility, stereopsis, and binocular alignment. Subjects with any previous eye surgery, structural ocular anomalies, amblyopia of either eye, ptosis, cataract, and nystagmus were excluded.

\section{Ophthalmic Examinations}

Visual acuity was measured using the Snellen chart. Cycloplegia was obtained by using compound tropicamide eye drops in children more than 6 years old, or atropine in 4-6year-old subjects. Objective and subjective refraction was conducted, and refractive status was recorded as spherical equivalent (SE) [spherical power + (cylindrical power)/2]. Flat keratometry (K1), steep keratometry (K2), central corneal thickness (CCT), white to white distance (WTW), pupil diameter (PD), anterior chamber depth (ACD), lens thickness (LT), and axial lengths (AL) were measured with OA2000 before the cycloplegia. Every subject had more than eight records taken at one time with a single OA-2000 measurement under uniform ambient light conditions. Only measurements with high accuracy $(\mathrm{SD}<0.04$ for keratometry, 0.02 for other parameters) and with level A were acceptable; 
A

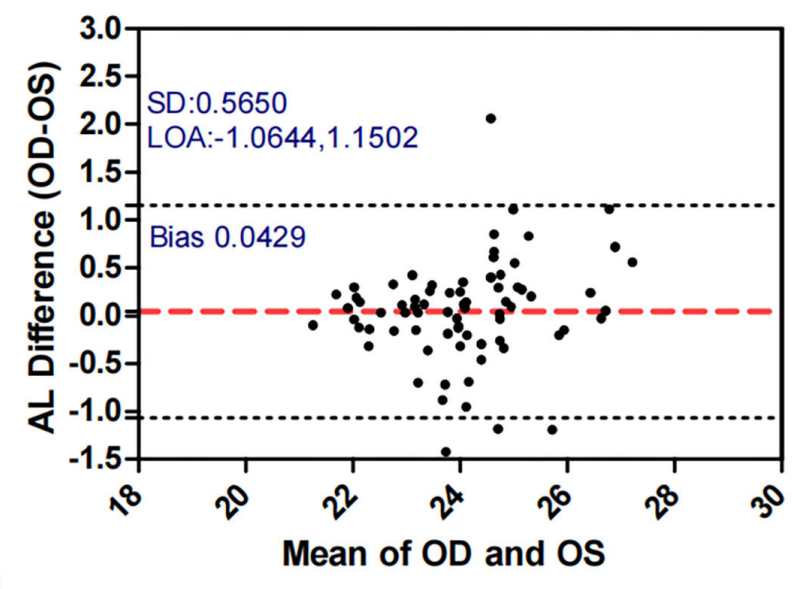

C

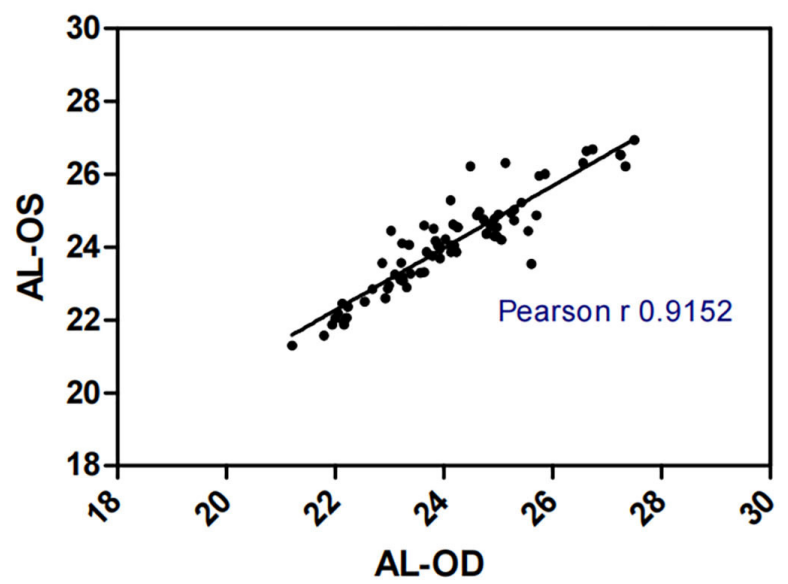

B

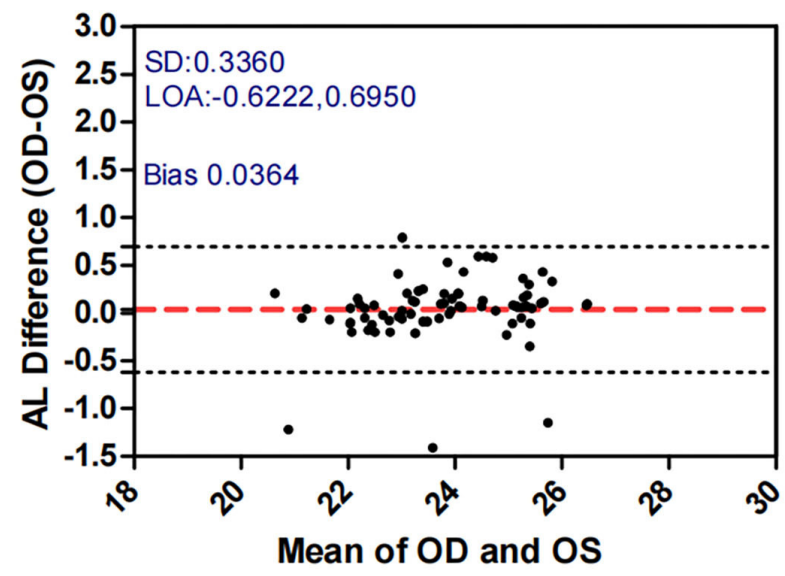

D

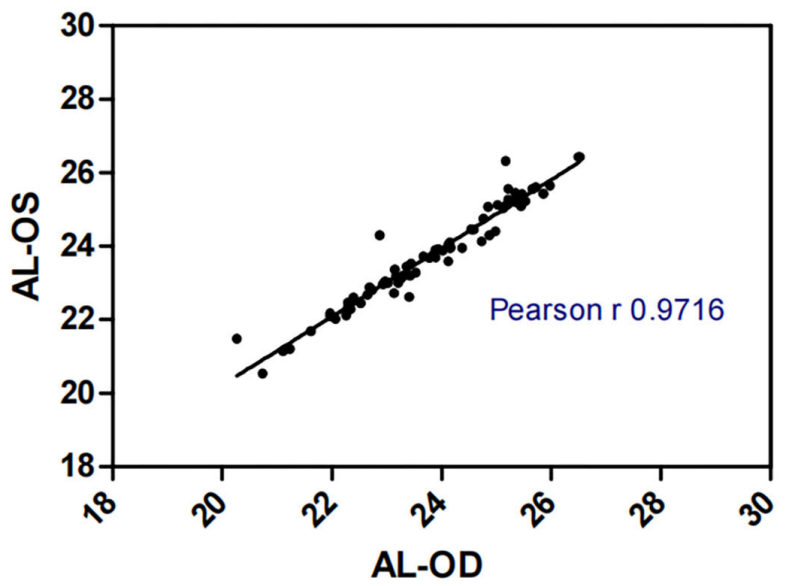

FIGURE 2 | Bland-Altman plots (A,B) and scatter plots (C,D) of AL in concomitant exotropia (A,C) and normal control participants (B,D) of the left and right eyes. OS, left eye; OD, right eye; $\mathrm{AL}$, axial length; LOA, 95\% limits of agreement.

otherwise, re-measurement was conducted. The absolute values of inter-eye differences for $\mathrm{SE}, \mathrm{PD}, \mathrm{ACD}, \mathrm{LT}$, and $\mathrm{AL}$ were recorded as AnisoSE, AnisoPD, AnisoACD, AnisoLT, and AnisoAL.

\section{Statistical Analysis}

The within-group inter-eye differences were analyzed by the intraclass correlation coefficient (ICC) and BlandAltman plot. ICCs were classified as excellent with values $>0.90$, good with values between 0.75 and 0.9 , moderate with values between 0.5 and 0.75 , and poor with values $<0.5$. An independent sample $t$-test was used to compare age, refractive status, ocular biometric measurements, and absolute values of inter-eye differences. MannWhitney $U$-test was used if equal variances were not assumed in Levene's Test. The $P<0.05$ was considered as statistically significant.

\section{RESULTS}

A total of 156 study subjects, including 79 concomitant exotropia patients (mean age \pm SD: $9.92 \pm 3.32$ ) and 77 control subjects (mean age \pm SD: $8.97 \pm 3.11$ ), were recruited with 32 females and 47 males in patients with concomitant exotropia, and 36 females and 41 males in control subjects. AnisoSE ( $t$ 2.369, P.019) was significantly larger in concomitant exotropia patients with ICC values of 0.877 in patients with concomitant exotropia and 0.943 in control subjects (Tables 1, 2). No statistically significant difference was found in gender and age.

\section{OA-2000 Findings}

No significant difference of either the left or the right eye was found in K1, K2, CCT, WTW, PD, ACD, LT, and AL between concomitant exotropia and the control subjects. AnisoAL, AnisoACD, AnisoPD, and AnisoLT were statistically significant in concomitant exotropia patients with unassumed 
A

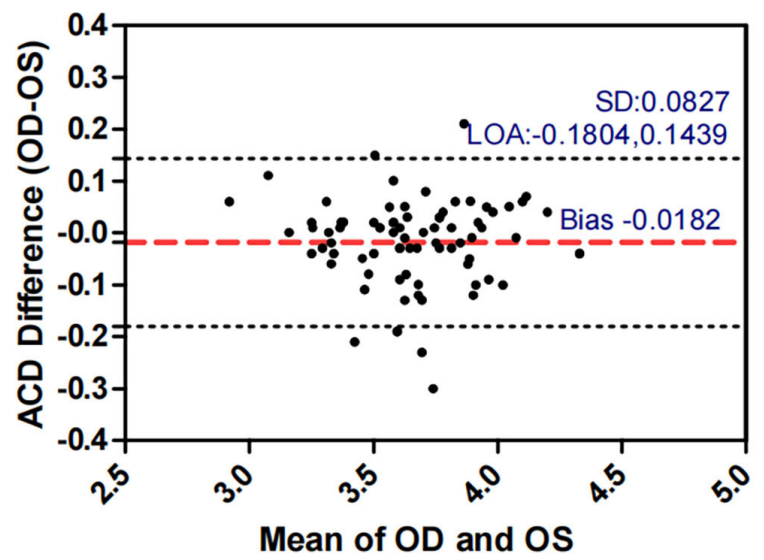

C

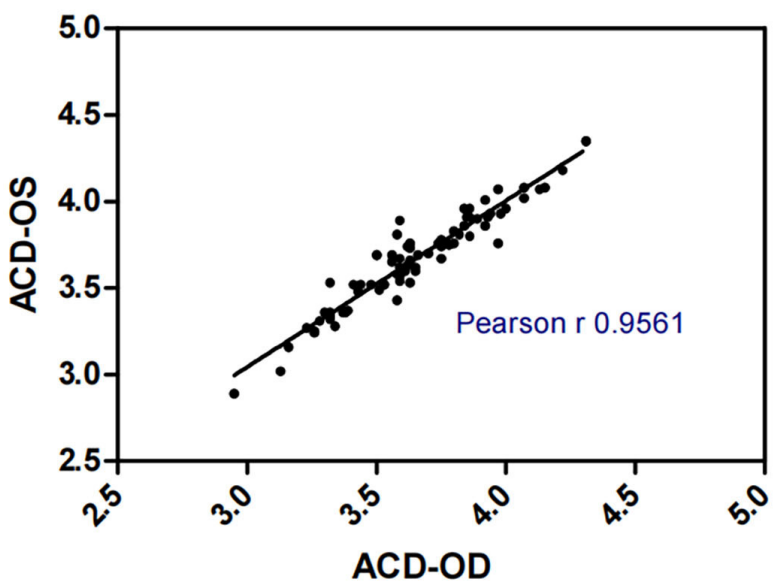

B

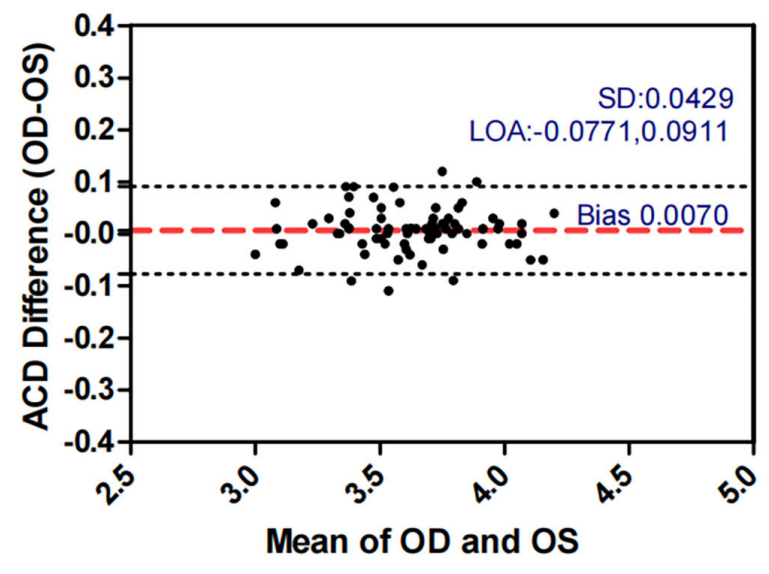

D

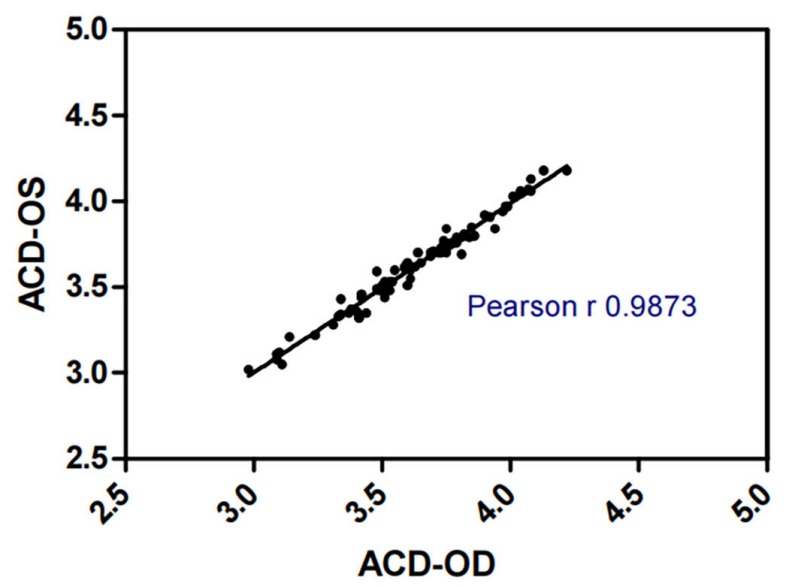

FIGURE 3 | Bland-Altman plots (A,B) and scatter plots (C,D) of ACD in concomitant exotropia (A,C) and normal control participants (B,D) of the left and right eyes. OS, left eye; OD, right eye; ACD, anterior chamber depth; LOA, 95\% limits of agreement.

equal variances. Therefore, these parameters were analyzed with a non-parametric Mann-Whitney $U$-test, finding that concomitant exotropia patients showed larger AnisoAL, AnisoACD, AnisoPD, and AnisoLT (Table 1).

Table 2 demonstrated the correlation coefficients of the ocular biometric parameters between the two eyes within concomitant exotropia and control subjects. AL (ICC $=0.914, r=0.915$ ), $\mathrm{ACD}(\mathrm{ICC}=0.955, r=0.956), \mathrm{PD}(\mathrm{ICC}=0.767, r=0.826)$, and LT (ICC $=0.924, r=0.924)$ showed good to excellent binocular correlation in concomitant exotropia patients. AL (ICC $=0.971$, $r=0.972), \mathrm{ACD}(\mathrm{ICC}=0.987, r=0.987), \mathrm{PD}(\mathrm{ICC}=0.944$, $r=0.945)$, and LT (ICC $=0.972, r=0.974)$ showed excellent binocular correlation in control subjects.

\section{Bland-Altman Plots Findings}

Figures 1-5 presented the Bland-Altman plots of the inter-eye differences in SE, AL, ACD, PD, and LT in concomitant exotropia $(\mathrm{A}, \mathrm{C})$ and also the normal control participants $(\mathrm{B}, \mathrm{D})$ of the left and right eyes. The ranges of agreement of the biometric parameters were listed in Table 3.

\section{DISCUSSION}

This study, for the first time, evaluated the ocular biometric features in patients with concomitant exotropia. In the present study, AnisoSE, AnisoAL, AnisoACD, AnisoPD, and AnisolT were significantly larger in patients with concomitant exotropia than in control subjects. SE, AL, ACD, PD, and LT showed good to excellent binocular correlation with high ICC values ranging from 0.767 to 0.955 in concomitant exotropia patients and a range from 0.943 to 0.987 in control participants.

\section{Interpretation of the Results}

Larger biometric inter-eye differences in patients with concomitant exotropia could be related to asymmetric binocular accommodative response. Previous studies reported the 
A

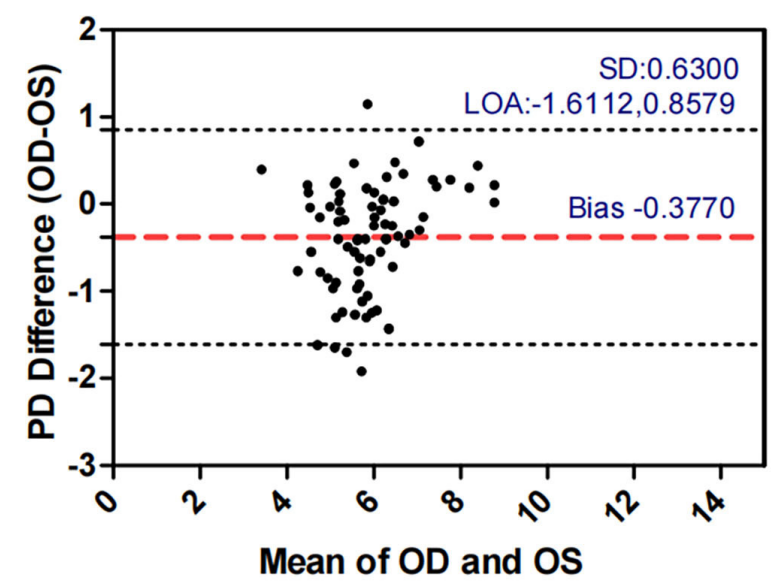

C

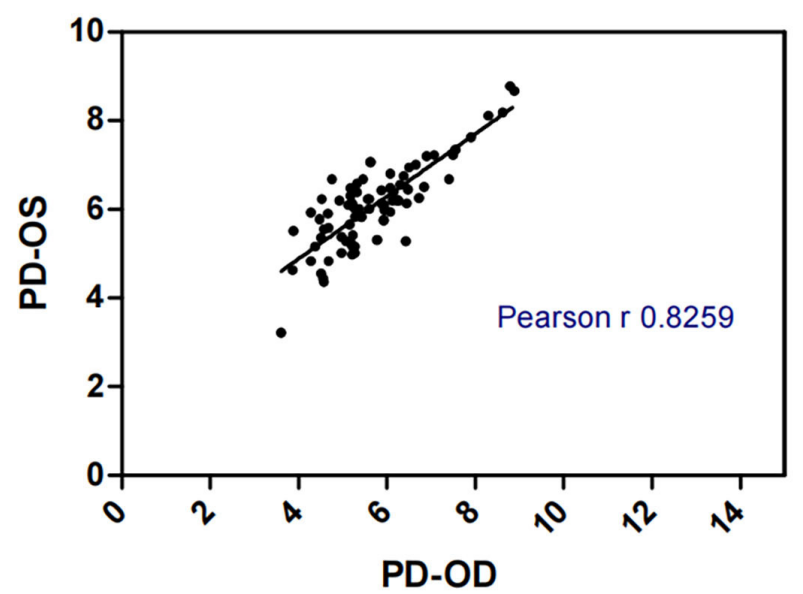

B

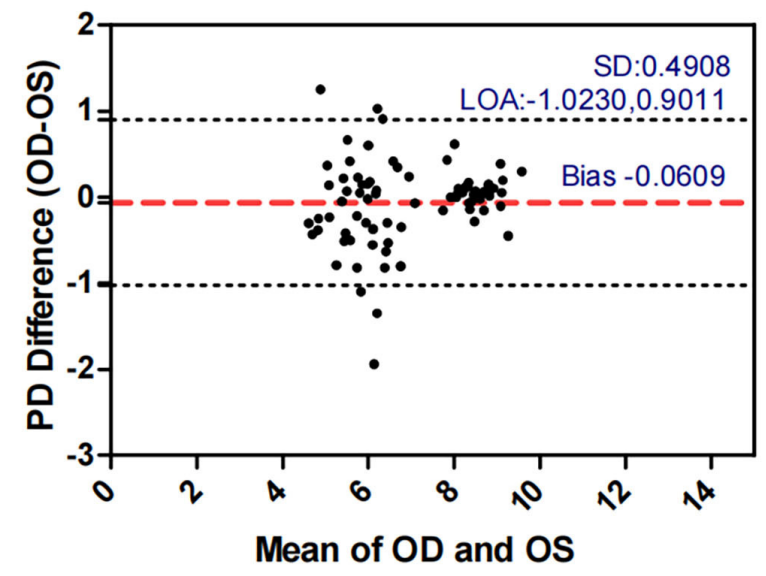

D

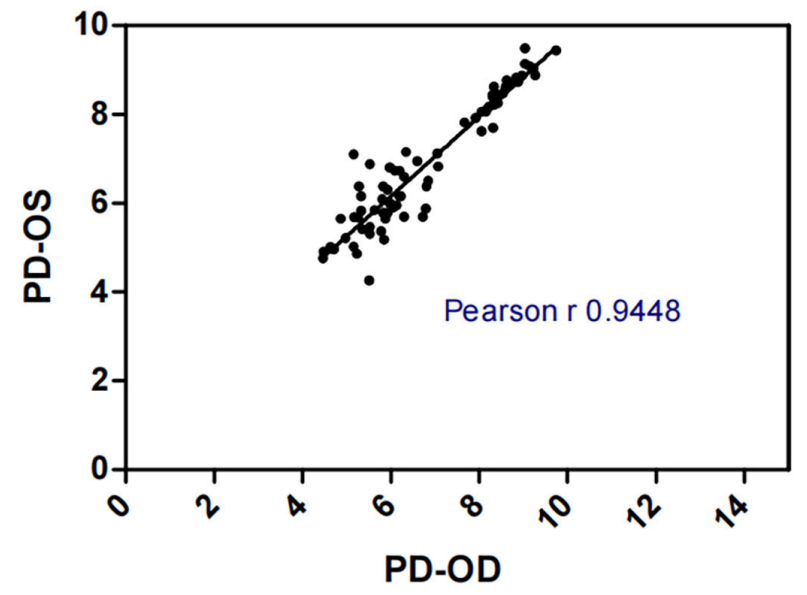

FIGURE 4 | Bland-Altman plots (A,B) and scatter plots (C,D) of PD in concomitant exotropia (A,C) and normal control participants (B,D) of the left and right eyes. OS, left eye; OD, right eye; PD, pupil diameter; LOA, 95\% limits of agreement.

asymmetric binocular accommodative response with decreased accommodation in the deviating eye (27) and increased accommodative loads for binocular fusion (28) in patients with concomitant exotropia. The decreased accommodation reflected a primary sensory loss over the central retinal region as a result of prolonged, early, and abnormal visual experience that is associated with abnormal interaction between the eyes, as well as the presence of strabismus and/or anisometropia (29).

Anisometropia had been reported to be associated with concomitant exotropia $(17,30-32)$. On the other hand, earlyonset concomitant exotropia could lead to anisometropia due to the disruption of the emmetropization process (11). In our study, anisometropia was significantly larger in concomitant exotropia patients (mean: 0.878) as compared to the control subjects (mean: 0.577). The SE in the control subjects showed excellent binocular correlation (ICC $=0.943, r=0.944$ ) and narrow range of agreement (Bias: $-0.097,95 \%$ limits of agreement:
$-1.760,1.566)$, while the binocular correlation in concomitant exotropia patients was weaker (ICC $=0.877, r=0.876$ ) with a wider range of agreement (Bias: 0.052, 95\% limits of agreement: $-2.462,2.567)$. Our study confirmed the association between anisometropia and concomitant exotropia.

Other biometric components such as AL, LT, and ACD are important in anisometropia (15), especially AL. AL as an indicator of myopic progression (33), had a strong correlation with anisometropia $(15,34)$. In our study, AnisoAL was significantly larger ( 0.395 vs 0.208$)$ with lower ICC (0.914 vs 0.971 ) in patients with concomitant exotropia.

LT and ACD, as the important factors in anisometropia, contribute to refractive error. During accommodation, the ACD decreases and the LT increases (35). Inter-eye differences in LT and ACD revealed the asymmetric accommodative response between two eyes. In our study, AnisoLT and AnisoACD were significantly larger $(0.060$ vs 0.031$)$ in 


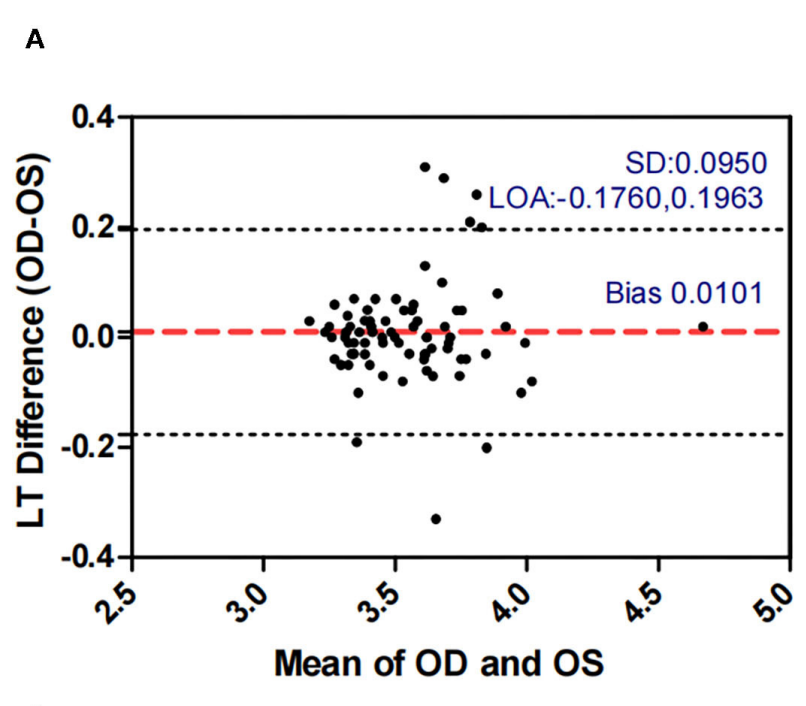

C

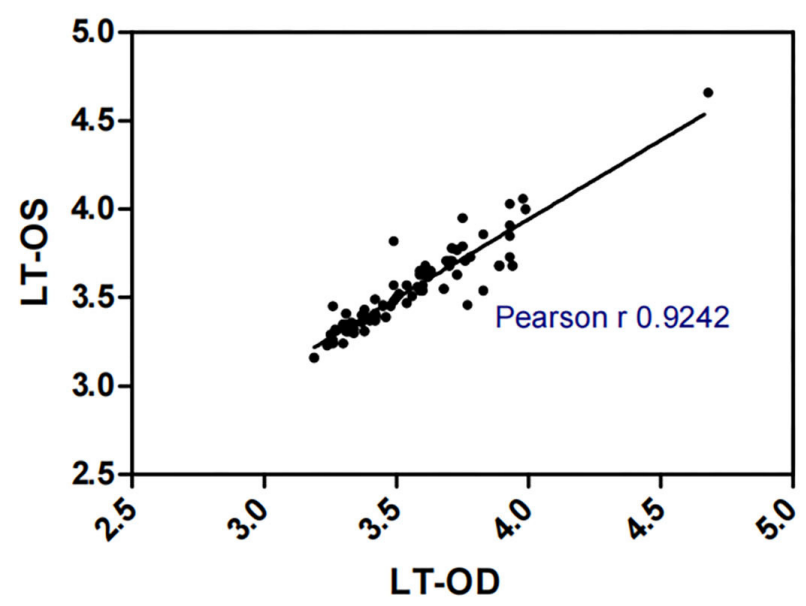

B

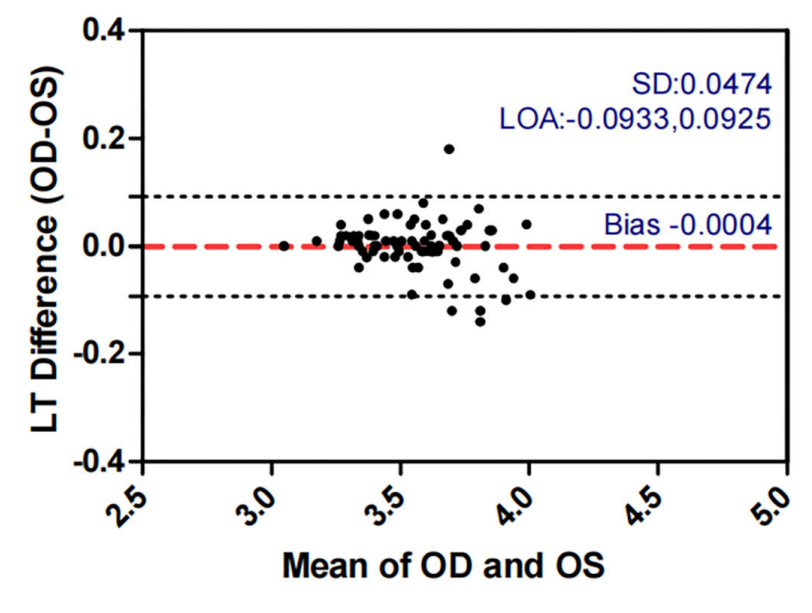

D

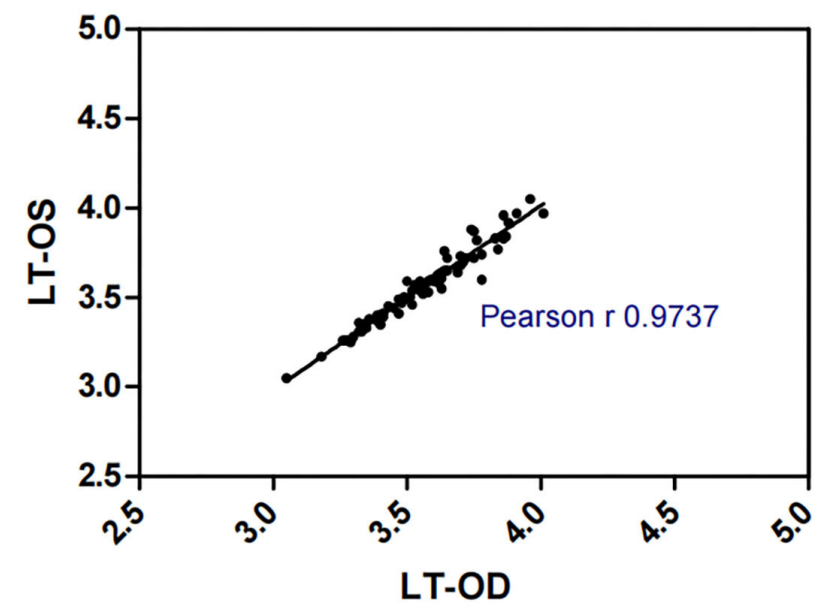

FIGURE $\mathbf{5}$ | Bland-Altman plots (A,B) and scatter plots (C,D) of LT in concomitant exotropia (A,C) and normal control participants $(\mathbf{B}, \mathbf{D})$ of the left and right eyes. OS, left eye; OD, right eye; LT, lens thickness; LOA, 95\% limits of agreement.

concomitant exotropia patients, indicating more inter-eye differences in accommodation. The control subjects had higher intra-class correlation (ICC $=0.972$ in LT, ICC $=0.987$ in ACD) and narrower range of agreement than patients with concomitant exotropia (ICC $=0.924$ in LT, ICC $=0.955$ in ACD).

Pupil diameter (PD) decreases with increasing age, retinal illumination, and near response. In our study, OA-2000 measurements were conducted under uniform ambient light conditions. The relationship between $\mathrm{PD}$ and concomitant exotropia is less frequently investigated in previous studies. In our study, concomitant exotropia patients showed larger AnisoPD (0.557 vs 0.340$)$. The PD in the control subjects had an excellent binocular correlation (ICC $=0.944, r=0.945$ ) and had a narrow range of agreement (Bias: $-0.061,95 \%$ limits of agreement: $-0.023,0.901)$. Yet, the binocular correlation in patients with concomitant exotropia was weaker $($ ICC $=0.767$, $r=0.826$ ), with a wider range of agreement (Bias: -0.377 , 95\% limits of agreement: $-1.611,0.858)$. Notably, Pearson's correlation was higher than ICC in patients with concomitant exotropia due to the difference in statistical analyses. The data were centered and scaled using a pooled mean and a standard deviation in ICC, but each variable was centered and scaled by its own mean and SD in the Pearson's correlation (36).

In conclusion, larger biometric inter-eye differences may be caused by concomitant exotropia itself, asymmetric binocular accommodation, anisometropia, or the interaction of these factors.

\section{STRENGTHS}

Unlike other technologies such as MRI, which has a superior soft-tissue contrast, evaluates both anatomic and physiologic 
TABLE 3 | Summary of the inter-ocular agreement of biometric parameters between concomitant exotropia and control participants.

\begin{tabular}{|c|c|c|c|c|c|c|c|c|}
\hline & \multicolumn{4}{|c|}{$\mathbf{X T}$} & \multicolumn{4}{|c|}{ NCP } \\
\hline SE (D) & $\begin{array}{c}0.0522 \pm \\
1.2828\end{array}$ & -2.4622 & 2.5666 & 5.0288 & $\begin{array}{c}-0.0974 \pm \\
0.8484\end{array}$ & -1.7603 & 1.5655 & 3.3258 \\
\hline $\mathrm{AL}(\mathrm{mm})$ & $\begin{array}{c}0.0429 \pm \\
0.5650\end{array}$ & -1.0644 & 1.1502 & 2.2146 & $\begin{array}{c}0.0364 \pm \\
0.3360\end{array}$ & -0.6222 & 0.6950 & 1.3172 \\
\hline PD (mm) & $\begin{array}{c}-0.3770 \pm \\
0.6300\end{array}$ & -1.6112 & 0.8579 & 2.4691 & $\begin{array}{c}-0.0609 \pm \\
0.4908\end{array}$ & -1.0230 & 0.9011 & 1.9241 \\
\hline LT (mm) & $\begin{array}{c}0.0101 \pm \\
0.0950\end{array}$ & -0.1760 & 0.1963 & 0.3723 & $\begin{array}{c}-0.0004 \pm \\
0.0474\end{array}$ & -0.0933 & 0.0925 & 0.1858 \\
\hline
\end{tabular}

XT, concomitant exotropia; NCP, normal control participants; SE, spherical equivalent; AL, axial length; ACD, anterior chamber depth; PD, pupil diameter; LT, lens thickness.

parameters simultaneously, and is particularly useful in rectus extraocular muscle evaluation (14), the optical biometry, such as OA 2000, has been proven to be more accurate and safer for ocular biometric measurements $(37,38)$. Only one measurement was taken for each subject, which was timesaving due to the high repeatability and reproducibility. By OA 2000 measurements, we revealed larger AnisoSE, AnisoAL, AnisoACD, AnisoLT, and AnisoPD in patients with concomitant exotropia. Subjects with larger AnisoSE, AnisoAL, AnisoACD, AnisolT, and AnisoPD were prone to develop concomitant exotropia. Advice should be given to the suspicious subjects to avoid or delay the development of concomitant exotropia.

\section{LIMITATIONS}

There were several limitations in this study. First, this was a cross-sectional study. Second, the study subjects were not randomly selected. Third, among all parameters measured by OA 2000, the measurements of PD should be adjusted manually by two technicians. Fourth, every subject with central fixation in our study had only one measurement, although more than 8 results were recorded. The measurement biases could not be avoided, even though OA-2000 shows high repeatability and reproducibility.

In summary, our study initially evaluated the ocular biometric features in patients with concomitant exotropia and found that AnisoSE, AnisoPD, AnisoAL, AnisoACD, and AnisoLT could contribute to concomitant exotropia. Our results will contribute to the etiology and management of concomitant exotropia.

\section{REFERENCES}

1. Beauchamp GR, Black BC, Coats DK, Enzenauer RW, Hutchinson AK, Saunders RA, et al. The management of strabismus in adults-I. Clinical characteristics and treatment.

\section{DATA AVAILABILITY STATEMENT}

The original contributions presented in the study are included in the article/supplementary material, further inquiries can be directed to the corresponding author/s.

\section{ETHICS STATEMENT}

The studies involving human participants were reviewed and approved by the Human Medical Research Ethics Committee of Joint Shantou International Eye Center of Shantou University and the Chinese University of Hong Kong. Written informed consent to participate in this study was provided by the participants' legal guardian/next of kin.

\section{AUTHOR CONTRIBUTIONS}

WG: study design, data collection, data analysis, and manuscript preparation. HC: data analysis and manuscript preparation. FY, CL, and SL: data collection. GW: study design and manuscript preparation. All authors contributed to the article and approved the submitted version.

\section{FUNDING}

This study was supported by Shantou Science and Technology Project (Grant No. 190629115260318), Guangdong Medical Research Foundation (CN) (Grant No. A2016514), Shantou Municipal Science and Technology Project (Grant No. 190917155269927), and 2020 Li Ka Shing Foundation Cross-Disciplinary Research Grant (Project Number: 2020LKSFG06B).

\section{$J$ AAPOS. (2003) 7:233-40. doi: 10.1016/S1091-8531(03)0} 0112-5

2. Nelson BA, Gunton KB, Lasker JN, Nelson LB, Drohan LA. The psychosocial aspects of strabismus in teenagers and adults and the impact of surgical correction. J AAPOS. (2008) 12:72-6 e1. doi: 10.1016/j.jaapos.2007.08.006 
3. Durnian JM, Noonan CP, Marsh IB. The psychosocial effects of adult strabismus: a review. Br J Ophthalmol. (2011) 95:4503. doi: $10.1136 /$ bjo. 2010.188425

4. Paysse EA, Steele EA, McCreery KM, Wilhelmus KR, Coats DK. Age of the emergence of negative attitudes toward strabismus. J AAPOS. (2001) 5:361-6. doi: 10.1067/mpa.2001.119243

5. Beauchamp GR, Black BC, Coats DK, Enzenauer RW, Hutchinson AK, Saunders RA, et al. The management of strabismus in adults-III. The effects on disability. J AAPOS. (2005) 9:455-59. doi: 10.1016/j.jaapos.2005.04.009

6. Van de Graaf ES, Borsboom G, van der Sterre GW, Felius J, Simonsz HJ, Kelderman H. Differences in quality-of-life dimensions of adult strabismus quality of life and amblyopia and strabismus questionnaires. Graefes Arch Clin Exp Ophthalmol. (2017) 255:1851-58. doi: 10.1007/s00417-017-3694-x

7. Menon V, Saha J, Tandon R, Mehta M, Khokhar S. Study of the psychosocial aspects of strabismus. J Pediatr Ophthalmol Strabismus. (2002) 39:2038. doi: 10.3928/0191-3913-20020701-07

8. Yang Y, Wang C, Gan Y, Jiang H, Fu W, Cao S, et al. Maternal smoking during pregnancy and the risk of strabismus in offspring: a meta-analysis. Acta Ophthalmol. (2019) 97:353-63. doi: 10.1111/aos.13953

9. Maconachie GD, Gottlob I, McLean RJ. Risk factors and genetics in common comitant strabismus: a systematic review of the literature. J Am Med Assoc Ophthalmol. (2013) 131:1179-86. doi: 10.1001/jamaophthalmol.2013.4001

10. Lithander J. Prevalence of amblyopia with anisometropia or strabismus among schoolchildren in the Sultanate of Oman. Acta Ophthalmol Scand. (1998) 76:658-62. doi: 10.1034/j.1600-0420.1998.760604.x

11. Smith E, Hung L, Arumugam B, Wensveen J, Chino Y, Harwerth R. Observations on the relationship between anisometropia, amblyopia and strabismus. Vision Res. (2017) 134:26-42. doi: 10.1016/j.visres.2017.03.004

12. Tang SM, Chan RY, Bin Lin S, Rong SS, Lau HH, Lau WW, et al. Refractive errors and concomitant strabismus: a systematic review and meta-analysis. $S c i$ Rep. (2016) 6:35177. doi: 10.1038/srep35177

13. Thouvenin D, Norbert O. Intraoperative assessment of medial rectus pulley location in strabismus. Eur J Ophthalmol. (2013) 23:13-8. doi: 10.5301/ejo.5000187

14. Hao R, Suh SY, Le A, Demer JL. Rectus extraocular muscle size and pulley location in concomitant and pattern exotropia. Ophthalmology. (2016) 123:2004-12. doi: 10.1016/j.ophtha.2016.05.053

15. Hashemi H, Khabazkhoob M, Emamian M, Shariati M, Abdolahinia $\mathrm{T}$, Fotouhi A. All biometric components are important in anisometropia, not just axial length. $B r \quad J$ Ophthalmol. (2013) 97:1586-91. doi: 10.1136/bjophthalmol-2013-303939

16. Tideman JWL, Polling JR, Vingerling JR, Jaddoe VWV, Williams C, Guggenheim JA, et al. Axial length growth and the risk of developing myopia in European children. Acta Ophthalmol. (2018) 96:301-09. doi: 10.1111/aos.13603

17. Huynh SC, Wang XY, Ip J, Robaei D, Kifley A, Rose KA, et al. Prevalence and associations of anisometropia and aniso-astigmatism in a population based sample of 6 year old children. Br J Ophthalmol. (2006) 90:597601. doi: 10.1136/bjo.2005.083154

18. Ferrer-Blasco T, Dominguez-Vicent A, Esteve-Taboada JJ, Aloy MA, Adsuara JE, Montes-Mico R. Evaluation of the repeatability of a swept-source ocular biometer for measuring ocular biometric parameters. Graefes Arch Clin Exp Ophthalmol. (2017) 255:343-9. doi: 10.1007/s00417-016-3555-Z

19. Savini G, Hoffer KJ, Balducci N, Barboni P, Schiano-Lomoriello D. Comparison of formula accuracy for intraocular lens power calculation based on measurements by a swept-source optical coherence tomography optical biometer. J Cataract Refract Surg. (2020) 46:27-33. doi: 10.1016/j.jcrs.2019.08.044

20. Liao X, Peng Y, Liu B, Tan QQ, Lan CJ. Agreement of ocular biometric measurements in young healthy eyes between IOLMaster 700 and OA-2000. Sci Rep. (2020) 10:3134. doi: 10.1038/s41598-020-59919-y

21. Aloy MÁ, Adsuara JE, Cerdá-Durán P, Obergaulinger M, Esteve-Taboada JJ, Ferrer-Blasco T, et al. Estimation of the mechanical properties of the eye through the study of its vibrational modes. PLoS ONE. (2017) 9:e0183892. doi: 10.1371/journal.pone.0183892

22. Esteve-Taboada JJ, Ferrer-Blasco T, Aloy MA, Adsuara JE, CerdaDuran P, Mimica P, et al. Ocular anatomic changes for different accommodative demands using swept-source optical coherence tomography: a pilot study. Graefes Arch Clin Exp Ophthalmol. (2017) 255:2399-406. doi: 10.1007/s00417-017-3801-z

23. Ferrer-Blasco T, Esteve-Taboada JJ, Monsalvez-Romin D, Aloy MA, Adsuara JE, Cerda-Duran P, et al. Ocular biometric changes with different accommodative stimuli using swept-source optical coherence tomography. Int Ophthalmol. (2019) 39:303-10. doi: 10.1007/s10792-017-0813-y

24. Ferrer-Blasco T, Esteve-Taboada JJ, Dominguez-Vicent A, Aloy MA, Adsuara JE, Mimica P, et al. Effect of contact lenses on ocular biometric measurements based on swept-source optical coherence tomography. Arq Bras Oftalmol. (2019) 82:129-35. doi: 10.5935/0004-2749.20190020

25. Reitblat O, Levy A, Kleinmann G, Assia EI. Accuracy of intraocular lens power calculation using three optical biometry measurement devices: the OA-2000, Lenstar-LS900 and IOLMaster-500. Eye. (2018) 32:124452. doi: 10.1038/s41433-018-0063-x

26. Huang J, Savini G, Hoffer KJ, Chen H, Lu W, Hu Q, et al. Repeatability and interobserver reproducibility of a new optical biometer based on sweptsource optical coherence tomography and comparison with IOLMaster. $\mathrm{Br} \mathrm{J}$ Ophthalmol. (2017) 101:493-98. doi: 10.1136/bjophthalmol-2016-308352

27. Shen PC, Zhang Y, Liu Y, Jiang J, Xu JJ, Lin HL, et al. Study on the difference of binocular accommodative response between patients with intermittent exotropia under different viewing condition. Zhonghua Yan Ke Za Zhi. (2018) 54:55-61. doi: 10.3760/cma.j.issn.0412-4081.2018.01.010

28. Ha SG, Jang SM, Cho YA, Kim SH, Song JS, Suh YW. Clinical exhibition of increased accommodative loads for binocular fusion in patients with basic intermittent exotropia. BMC Ophthalmol. (2016) 16:77. doi: $10.1186 / s 12886-016-0260-y$

29. Ciuffreda KJ, Hokoda SC, Hung GK, Semmlow JL. Accommodative stimulus/response function in human amblyopia. Doc Ophthalmol. (1984) 56:303-26. doi: 10.1007/BF001 55676

30. Multi-ethnic Pediatric Eye Disease Study G. Prevalence of amblyopia and strabismus in African American and Hispanic children ages 6 to 72 months the multi-ethnic pediatric eye disease study. Ophthalmology. (2008) 115:122936 e1. doi: 10.1016/j.ophtha.2007.08.001

31. Yekta A, Hashemi H, Norouzirad R, Ostadimoghaddam $\mathrm{H}$, Nabovati P, Dadbin $\mathrm{N}$, et al. The prevalence of amblyopia, strabismus, and ptosis in schoolchildren of Dezful. Eur J Ophthalmol. (2017) 27:109-12. doi: 10.5301/ejo.50 00795

32. Pan CW, Chen X, Zhu H, Fu Z, Zhong H, Li J, et al. School-based assessment of amblyopia and strabismus among multiethnic children in rural China. Sci Rep. (2017) 7:13410. doi: 10.1038/s41598-017-1 3926-8

33. Huang J, Wen D, Wang Q, McAlinden C, Flitcroft I, Chen $\mathrm{H}$, et al. Efficacy comparison of 16 interventions for myopia control in children: a network meta-analysis. Ophthalmology. (2016) 123:697-708. doi: 10.1016/j.ophtha.2015. 11.010

34. Pärssinen $\mathrm{O}$, Kauppinen $\mathrm{M}$. Anisometropia of spherical equivalent and astigmatism among myopes: a 23-year follow-up study of prevalence and changes from childhood to adulthood. Acta ophthalmologica. (2017) 95:518-24. doi: 10.1111/aos. 13405

35. Schachar RA, Mani M, Schachar IH. Image registration reveals central lens thickness minimally increases during accommodation. Clin Ophthalmol. (2017) 11:1625-36. doi: 10.2147/OPTH.S1 44238

36. Fang D, Tang FY, Huang H, Cheung CY, Chen H. Repeatability, interocular correlation and agreement of quantitative swept-source optical coherence tomography angiography macular metrics in healthy subjects. Br J Ophthalmol. (2019) 103:415-20. doi: 10.1136/bjophthalmol-2018-3 11874

37. Guimaraes de Souza R, Montes de Oca I, Esquenazi I, Al-Mohtaseb Z, Weikert MP. Updates in biometry. Int Ophthalmol Clin. (2017) 57:115-24. doi: 10.1097/IIO.0000000000 000175

38. Savini G, Hoffer KJ, Schiano-Lomoriello D. Agreement between lens thickness measurements by ultrasound immersion biometry and optical 
biometry. J Cataract Refract Surg. (2018) 44:1463-68. doi: 10.1016/j.jcrs.2018. 07.057

Conflict of Interest: The authors declare that the research was conducted in the absence of any commercial or financial relationships that could be construed as a potential conflict of interest.

Publisher's Note: All claims expressed in this article are solely those of the authors and do not necessarily represent those of their affiliated organizations, or those of the publisher, the editors and the reviewers. Any product that may be evaluated in this article, or claim that may be made by its manufacturer, is not guaranteed or endorsed by the publisher.

Copyright $\odot 2022$ Gong, Chen, Yang, Lin, Li and Wang. This is an open-access article distributed under the terms of the Creative Commons Attribution License (CC $B Y)$. The use, distribution or reproduction in other forums is permitted, provided the original author(s) and the copyright owner(s) are credited and that the original publication in this journal is cited, in accordance with accepted academic practice. No use, distribution or reproduction is permitted which does not comply with these terms. 